\title{
The voltage-mode first order universal filter using single voltage differencing differential input buffered amplifier with electronic controllability
}

\author{
Danupat Duangmalai ${ }^{1}$, Peerawut Suwanjan ${ }^{2}$ \\ ${ }^{1}$ Department of Electronics, Faculty of Industrial Technology, Nakhon Phanom University, Mueang, Thailand \\ ${ }^{2}$ Department of Engineering Education, School of Industrial Education and Technology, King Mongkut's Institute of Technology \\ Ladkrabang, Bangkok, Thailand
}

\begin{abstract}
Article Info
Article history:

Received Jun 2, 2021

Revised Sep 9, 2021

Accepted Oct 6, 2021

Keywords:

Electronic control

First-order filter

LT1228

Voltage differencing

differential input bufferes

amplifier

ABSTRACT

In this research contribution, the electronically tunable first-order universal filter employing a single voltage differencing differential input buffered amplifier (VD-DIBA) (constructed from two commercially available integrated circuit (IC): the operational transconductance amplifier, IC number LT1228, and the differential voltage input buffer, IC number AD830), one capacitor and two resistors. The features of the designed first order universal filter are as follows. Three voltage-mode first-order functions, low-pass (LP), all-pass (AP) and high-pass (HP) responses are given. The natural frequency $\left(\omega_{0}\right)$ of the presented configuration can be electronically adjusted by setting the DC bias current. Moreover, the voltage gain of the LP and HP filters can be controllable. The phase responses of an AP configuration can be varied from $0^{\circ}$ to $-180^{\circ}$ and $180^{\circ}$ to $0^{\circ}$. The power supply voltages were set at $\pm 5 \mathrm{~V}$. Verification of the theoretically described performances of the introduced electronically tunable universal filter was proved by the PSpice simulation and experiment.
\end{abstract}

Voltage model
This is an open access article under the CC BY-SA license.

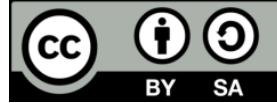

\section{Corresponding Author:}

Danupat Duangmalai

Department of Electronics, Faculty of Industrial Technology, Nakhon Phanom University

124 Moo.12, Chayangkul Road, Khamthao Sub-district, Muang District, Nakhon Phanom, Thailand

Email: danupat@npu.ac.th

\section{INTRODUCTION}

The filters are very useful and widely used in electronics, instrumentation, and communication systems especially in signal and image processing systems [1]. Filter can be categorized by its different types of filters such as, passive filter and active filter. The realization of analog active filters employing the active building block in which one of the most popular active filters is single-input multiple-output (SIMO) filtering configuration [2] or multiple-input single-output (MISO) filtering configuration [3]. The components of actives filter can be minimized by employing a single active building blocks that can be designed in both voltage-mode and current-mode configurations.

As stated above, the universal active filters give many advantages. So, there are many active filters that have been realized, implemented, and demonstrated. These active filters emphasis on employing the active building block which exhibit many advantages such as their cut-off frequency tuning, several filter functions, cascadability, low-voltage (LV), and low-power (LP) [4]-[6]. The first-order universal filter is a very interesting circuit in design. The universal first-order filter using electronically controllable active elements, namely operational transconductance amplifiers (OTA) is proposed in [7], [8]. The proposed filter in [7], consists of two OTAs and two passive elements (one resistor and one capacitor). In study [8], it 
comprises two OTAs as active elements with four resistors and one capacitor. The current mode first order filtering configuration employing the second-generation current conveyor (CCII) is presented in [9], [10]. In study [9], it consists of two CCIIs, four resistors, and one capacitor. The first order filter in [9] was also modified to reduce two resistors by employing second generation current controlled current conveyors (CCCIIs). In [10], the single CCII based first-order allpass section is introduced. This first order configuration is very simple, but the filtering parameter cannot be electronically adjusted. The first order filter employing the differential voltage current conveyor (DVCC) is proposed in [11]-[13]. In studies [11], [12], authors consist of single DVCC and passive devices. In [13], this filter is constructed from two DVCCs, single grounded capacitor and single resistor. However, the filtering parameters of theses first order universal filter [11]-[13] are not electronically tuned. The DDCC is employed to design the first order allpass filter [14]. This filter is very simple consisting of one DDCC, one resistor and grounded capacitor, but the cut-off frequency and adjustment of phase response is not electronically controlled. In [15], it consists of one differential difference current conveyor transconductance amplifier (DDCCTA) and one capacitor. In this filtering circuit, the cut-off frequency and phase response [15] are electronically tuned, but the voltage output node is not low impedance. The fully differential current conveyor (FDCCII) [16] is used to design the first order filter in [16]. It consists of single FDCCII, one grounded resistor and one grounded capacitor. However, the filtering parameters of theses first order filter [16] are not electronically tuned. The voltage differencing transconductance amplifier (VDTA) is employed to design the first order in [17]. This circuit is constructed from a single VDTA and a capacitor, but its voltage output node is not low impedance. Additionally, the performances of the proposed filters in [7]-[17] are not verified experimentally.

According to the benefit of using an active building block to design the circuit, the voltage differencing differential input buffered amplifier (VD-DIBA) [18] is introduced as a versatile active device. It has interesting applications with neat features, especially electronic controllability, high input voltage terminal, low output voltage terminal, and high output current terminal. VD-DIBA can be used to realize high-performance analog circuits [19]-[23].

The purpose of this contribution is to realize the first-order universal filter using a single VD-DIBA, one capacitor, and two capacitors. The VD-DIBA is implemented from two commercial integrated circuit (ICs): the operational transconductance amplifier, IC number LT1228, and the differential-input buffer, IC number AD830. The proposed circuit can provide three filtering voltage-mode functions: LP, HP, and AP responses. Verification of the described functionality of the presented filter is illustrated by PSpice simulation and experiment.

\section{PRINCIPLE OF OPERATION}

There are three sub-sections that will be described in this section which are the ideal characteristics of the active element, VD-DIBA, detail of proposed filter, and non-ideal study of the presented first-order filter.

\subsection{VD-DIBA overview}

The detail of the ideal VD-DIBA will be provided in this sub-section. The VD-DIBA has five input and output terminal with a low impedance at the $w$ output voltage terminal, a high impedance at the $z$ output current terminal and high impedance at the $v_{+}, v_{-}$, and $v$ input voltage terminals. The transconductance $\left(g_{m}\right)$ at $z$ terminal can be electronically tuned by the bias current. The different voltage at $z$ and $v$ terminal is transfered to the $w$ terminal with unity gain. The electrical circuit representation of VDDIBA is illustrated in Figure 1(a) and the equivalent diagram of VD-DIBA is depicted in Figure 1(b). The behavior of the voltage and the current at the input and output ports of VD-DIBA are given by (1).

$$
\left[\begin{array}{c}
I_{V_{+}} \\
I_{V_{-}} \\
I_{z} \\
I_{v} \\
V_{w}
\end{array}\right]=\left[\begin{array}{ccccc}
0 & 0 & 0 & 0 & 0 \\
0 & 0 & 0 & 0 & 0 \\
g_{m} & -g_{m} & 0 & 0 & 0 \\
0 & 0 & 0 & 0 & 0 \\
0 & 0 & 1 & -1 & 0
\end{array}\right]\left[\begin{array}{c}
V_{+} \\
V_{-} \\
V_{z} \\
V_{v} \\
I_{w}
\end{array}\right]
$$

\subsection{The proposed voltage-mode first order filter using single VD-DIBA}

To design the proposed filter in this paper, the VD-DIBA is employed as an active building block. This design has emphasized the use of single VD-DIBA to achieve the LP, HP, and AP first-order filtering functions with electronic control of the filtering parameters, natural frequency, and phase response. With these specifications, the design of the proposed universal first-order filer is achieved in Figure 2 . It is constructed from a single VD-DIBA, one capacitor, and two resistors with three input voltage nodes, $\mathrm{V}_{\text {inl }}$, 
$\mathrm{V}_{\text {in2 }}, \mathrm{V}_{\mathrm{in} 3}$ and single output voltage node $\mathrm{V}_{\mathrm{o}}$. The output voltage node is at the $\mathrm{w}$ terminal which exhibits low impedance. With this configuration, the proposed first-order filter can be cascaded to other circuits without the need for an additional voltage buffer. Based on the ideal properties of VD-DIBA mentioned in section 2.1, the output voltage, $\mathrm{V}_{\mathrm{o}}$ is obtained Figure 2 .

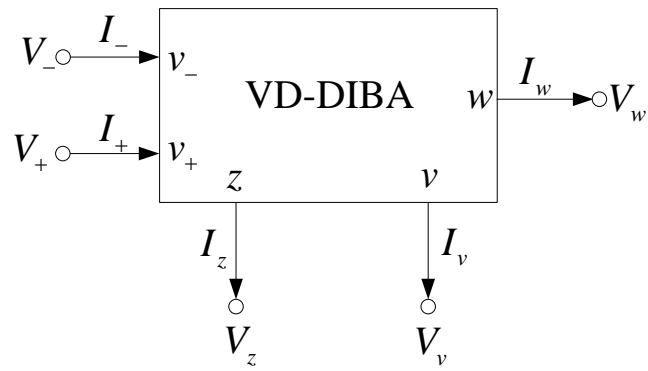

(a)

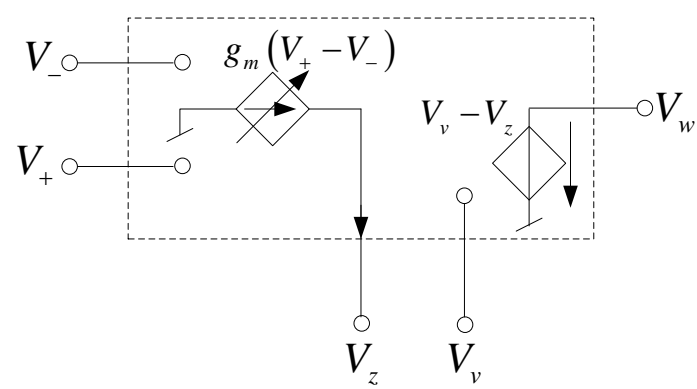

(b)

Figure 1. The VD-DIBA on (a) electrical circuit symbol and (b) equivalent circuit

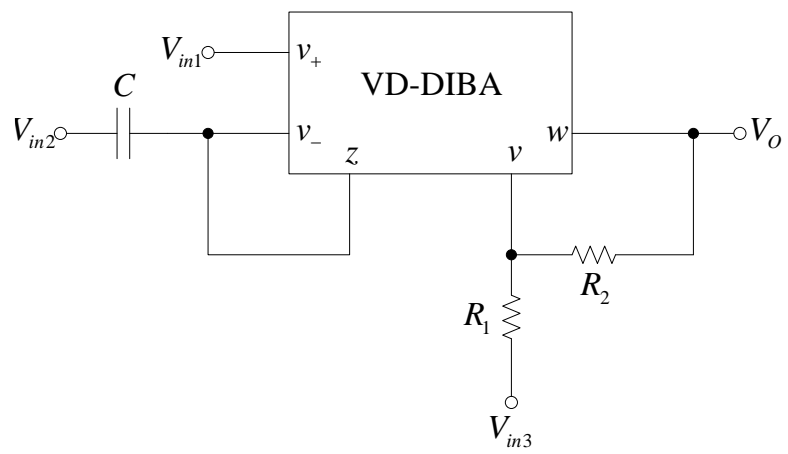

Figure 2. The proposed first order filter using single VD-DIBA

$$
V_{O}=\frac{\left(s C+g_{m}\right) V_{i n 3}-k g_{m} V_{i n 1}-k s C V_{i n 2}}{s C+g_{m}}
$$

Considering, the output voltage shown in (2), the transfer function in voltage-mode system for first order filtering LP and HP responses can be selected as follow:

- When $V_{i n 1}=V_{i n}$ and $V_{i n 2}=V_{i n 3}=0$ (grounded), the transfer function in voltage-mode form of LP response can be expressed in (3),

$$
\frac{V_{O}}{V_{i n}}=\frac{-k g_{m}}{s C+g_{m}}
$$

- When $V_{i n 2}=V_{i n}$ and $V_{i n 1}=V_{i n 3}=0$, the transfer function in voltage-mode form of HP can be expressed in (4)

$$
\frac{V_{O}}{V_{i n}}=\frac{-k s C}{s C+g_{m}}
$$

when $k=\frac{R_{2}}{R_{1}}+1$. It is found that the voltage gain of the proposed filter for LP and HP filters can be controllable.

Considering, the output voltage shown in (2), if $\mathrm{R}_{1}=\mathrm{R}_{2}(\mathrm{k}=2)$, the transfer function in voltage-mode form for first order filtering AP responses can be selected as follow: 
- When $V_{i n 2}=V_{i n 3}=V_{i n}$ and $V_{i n 1}=0$ the voltage transfer function of $\mathrm{AP}_{\mathrm{N}}$ can be expressed in (5).

$$
\frac{V_{O}}{V_{\text {in }}}=\left(\frac{g_{m}-s C}{g_{m}+s C}\right)
$$

- When $V_{i n 1}=V_{i n 3}=V_{i n}$ and $V_{i n 2}=0$ the transfer function in voltage-mode form of $\mathrm{AP}_{\mathrm{P}}$ can be expressed in (6).

$$
\frac{V_{O}}{V_{\text {in }}}=-\left(\frac{g_{m}-s C}{g_{m}+s C}\right)
$$

It is found from (5) and (6) that the voltage gain of the proposed filter for AP responses is a unity gain. The phase response of the LP and the HP can be expressed in (7) and (8), respectively.

$$
\begin{aligned}
& \theta_{L P}=180-\tan ^{-1}\left(\frac{\omega C}{g_{m}}\right) \\
& \theta_{H P}=\tan ^{-1}\left(\frac{g_{m}}{\omega C}\right)-180
\end{aligned}
$$

The phase response of the LP in (7) can be varied from $180^{\circ}$ to $0^{0}$, and in (8) the phase response of the HP can be varied from $-90^{\circ}$ to $-180^{\circ}$. The phase responses of the all-pass functions can be expressed as (9),

$$
\theta_{A P_{N}}=-2 \tan ^{-1}\left(\frac{\omega C}{g_{m}}\right)
$$

and

$$
\theta_{A P_{P}}=180-2 \tan ^{-1}\left(\frac{\omega C}{g_{m}}\right)
$$

The phase response of $\mathrm{AP}_{\mathrm{N}}$ in (9) can be varied from $0^{0}$ to $-180^{\circ}$, and in (10) the phase response of $\mathrm{AP}_{\mathrm{P}}$ can be varied from $180^{\circ}$ to $0^{0}$. Moreover, the phase responses can be electronically controlled by adjusting only a single bias current. Additionally, the proposed filer can realize three first-order responses which are LP HP and AP functions in the same configuration. The voltage mode transfer functions in (4)-(6) reveal that the natural frequency of the filter is shown in (11).

$$
\omega_{0}=\frac{g_{m}}{C}
$$

From (11), the natural frequency is electronically controllable.

\subsection{Effect of VD-DIBA non-ideal properties}

The analysis of the effect of non-idealities of VD-DIBA to filtering performances will be shown in this section. These effects include the tracking error gains and parasitic elements. First analysis will be focused on the voltage tracking error gains in VD-DIBA. The VD-DIBA properties with voltage tracking error gains are given in (12).

$$
\left[\begin{array}{l}
I_{V_{+}} \\
I_{V_{-}} \\
I_{z} \\
I_{v} \\
V_{w}
\end{array}\right]=\left[\begin{array}{ccccc}
0 & 0 & 0 & 0 & 0 \\
0 & 0 & 0 & 0 & 0 \\
g_{m} & -g_{m} & 0 & 0 & 0 \\
0 & 0 & 0 & 0 & 0 \\
0 & 0 & \beta_{z} & -\beta_{v} & 0
\end{array}\right]\left[\begin{array}{l}
V_{+} \\
V_{-} \\
V_{z} \\
V_{v} \\
I_{w}
\end{array}\right]
$$

Where $\beta_{z}$ and $\beta_{v}$ are the voltage tracking error gains from $z$ and $v$ terminals to $w$ terminal. Considering these tracking error gains, the output voltage of the electronically controllable first order filter in Figure 2 is given as in (13). 


$$
V_{O}^{*}=\frac{\left(s C+g_{m}\right) \beta_{v} V_{i n 3}-k g_{m} \beta_{z} V_{i n 1}-k s C \beta_{z} V_{i n 2}}{s C \beta_{v}+g_{m} \beta_{v}}
$$

From (13), the non-ideal magnitudes with the voltage tracking error gains for each transfer functions in voltage-mode form are obtained as (14), (15), and (16),

$$
\begin{aligned}
& \left|T_{L P}{ }^{*}\right|=\frac{|k|}{\sqrt{\left(\frac{\beta_{v}}{\beta_{z}}\right)^{2}+\left(\frac{\beta_{v} \omega C}{\beta_{z} g_{m}}\right)^{2}}} \\
& \left|T_{H P}{ }^{*}\right|=\frac{|k|}{\sqrt{\left(\frac{\beta_{v}}{\beta_{z}}\right)^{2}+\left(\frac{\beta_{v} g_{m}}{\beta_{z} \omega C}\right)^{2}}} \\
& \left|T_{A P_{N}}{ }^{*}\right|=\frac{\sqrt{\left(\beta_{v} g_{m}\right)^{2}+\left(\beta_{v} \omega C-\beta_{z} k \omega C\right)^{2}}}{\sqrt{\left(\beta_{v} g_{m}\right)^{2}+\left(\beta_{v} \omega C\right)^{2}}}
\end{aligned}
$$

and

$$
\left|T_{A P_{P}}{ }^{*}\right|=\frac{\sqrt{\left(g_{m}\left(\beta_{v}-\beta_{z} k\right)\right)^{2}+\left(\beta_{v} \omega C\right)^{2}}}{\sqrt{\left(\beta_{v} g_{m}\right)^{2}+\left(\beta_{v} \omega C\right)^{2}}}
$$

The phase responses with the voltage tracking error gains for the all-pass function can be obtained as (18), (19),

$$
\begin{aligned}
& \theta_{A P_{N}}{ }^{*}=180-\tan ^{-1} \frac{\omega\left(\beta_{v} C-\beta_{z} 2 C\right)}{\beta_{v} g_{m}}-\tan ^{-1} \frac{\omega C}{g_{m}} \\
& \theta_{A P_{P}}{ }^{*}=180-\tan ^{-1} \frac{\omega C \beta_{v}}{g_{m}\left(\beta_{v}-2 \beta_{z}\right)}-\tan ^{-1} \frac{\omega C}{g_{m}}
\end{aligned}
$$

Second analysis will be focused on the parasitic elements in VD-DIBA. The representation of the proposed electronically controllable first order universal filter with parasitic effect is depicted in Figure 3.

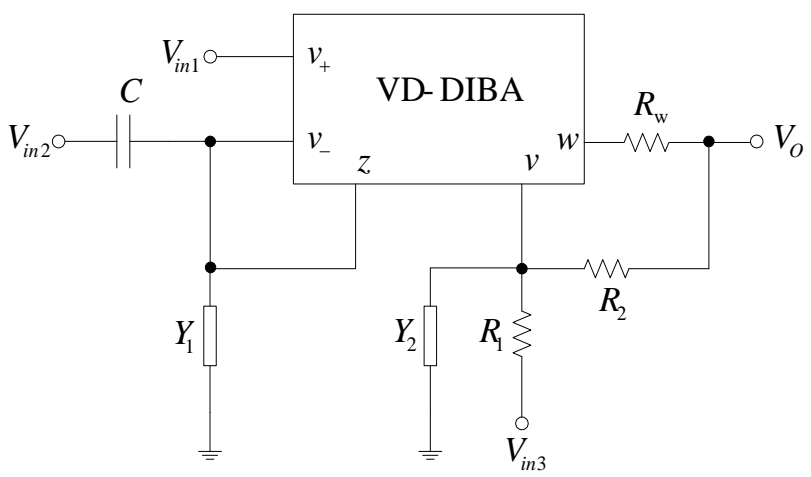

Figure 3. The VD-DIBA model with parasitic impedances

Considering in Figure 3, the admittances Y1 and Y2 are as (20):

$$
Y_{1}=s\left(C_{z}+C_{-}\right)+G_{z}+G_{-}
$$




$$
Y_{2}=s C_{v}+G_{v}
$$

Considering the effect of the parasitic impedance, the output voltage can be expressed as (22),

$$
V_{o}^{* *}=\left[\frac{V_{3} R_{2}}{Y_{2} R_{1} R_{2}+R_{2}}\right]-\left[\frac{V z\left(Y_{2} R_{1} R_{2}+R_{1}+R_{2}\right)}{Y_{2} R_{1} R_{2}+R_{2}}\right]\left(\frac{R_{2}}{R_{2}+R_{w}}\right)
$$

If the operational frequency $f_{o p} \ll \frac{1}{2 \pi R_{p} C_{v}}\left(Y_{2} \cong 0\right)$ and $C_{M}=\left(C+C_{z}+C_{-}\right), g_{m_{M}}=\left(g_{m}+G_{z}+G_{-}\right)$, the output voltage in (22) is modified to (23),

$$
V_{o}^{* * *}=\frac{V_{3}\left(s C_{M}+g_{m_{M}}\right)-V_{2} k s C-V_{1} k g_{m}}{s C_{M}+g_{m_{M}}}\left(\frac{R_{2}}{R_{2}+R_{w}}\right)
$$

From (23), the non-ideal magnitudes with the parasitic effects for each voltage transfer functions are given by (24), (25), and (26),

$$
\begin{aligned}
& \left|T_{L P}^{* * *}\right|=\frac{|k|}{\sqrt{\left(\frac{g_{m_{M}}}{g_{m}}\right)^{2}+\left(\frac{\omega C_{M}}{g_{m}}\right)^{2}}}\left(\frac{R_{2}}{R_{2}+R_{w}}\right) \\
& \left|T_{H P}^{* * *}\right|=\frac{|k|}{\sqrt{\left(\frac{C_{M}}{C}\right)^{2}+\left(\frac{g_{m_{M}}}{\omega C_{M}}\right)^{2}}}\left(\frac{R_{2}}{R_{2}+R_{w}}\right) \\
& \left|T_{A P_{N}}{ }^{* * *}\right|=\frac{\sqrt{\left(g_{m_{M}}\right)^{2}+\omega\left(C_{M}-2 C\right)^{2}}}{\sqrt{\left(g_{m_{M}}\right)^{2}+\left(\omega C_{M}\right)^{2}}}\left(\frac{R_{2}}{R_{2}+R_{w}}\right)
\end{aligned}
$$

and

$$
\left|T_{A P_{P}}{ }^{* * *}\right|=\frac{\sqrt{\left(g_{m_{M}}-2 g_{m}\right)^{2}+\left(\omega C_{M}\right)^{2}}}{\sqrt{\left(g_{m_{M}}\right)^{2}+\left(\omega C_{M}\right)^{2}}}\left(\frac{R_{2}}{R_{2}+R_{w}}\right)
$$
and (30),

The phase responses with parasitic effects for each filtering functions can be expressed as (28), (29),

$$
\begin{aligned}
& \theta_{L P}{ }^{* * *}=180-\tan ^{-1}\left(\frac{\omega C_{M}}{g_{m_{M}}}\right) \\
& \theta_{H P}{ }^{* * *}=\tan ^{-1}\left(\frac{g_{m_{M}}}{\omega C_{M}}\right)-180 \\
& \theta_{A P_{N}}{ }^{* * *}=-\tan ^{-1} \frac{\omega\left(C_{M}-2 C\right)}{g_{m_{M}}}-\tan ^{-1} \frac{\omega C_{M}}{g_{m_{M}}}
\end{aligned}
$$

and

$$
\theta_{A P_{P}}^{* * *}=180-\tan ^{-1} \frac{\omega C_{M}}{\left(g_{m_{M}}-2 g_{m}\right)}-\tan ^{-1} \frac{\omega C_{M}}{g_{m_{M}}}
$$




\section{SIMULATION AND EXPERIMENTAL RESULTS}

In this section, the Pspice program and experiment were used to evaluate the functionality of the presented voltage-mod first-order universal filter. The VD-DIBA used in the proposed circuit was constructed from two commercial ICs as shown in Figure 4. It comprises two basic blocks: the OTA IC number LT1228 [24], and the differential-input buffer, IC number AD830 [25] as the output stage. The following parameters were used: the resistor $R_{1}=R_{2}=1 \mathrm{k} \Omega$, the capacitor $C=1 \mathrm{nF}$, the transconductance $g_{m}=1 \mathrm{mS}$, the bias current $I_{B}=100 \mu \mathrm{A}$. The power supply voltages were set at $V_{D D}=-V_{S S}=5 \mathrm{~V}$. The theoretical natural frequency from above elemnt values is $f_{o}=159.15 \mathrm{kHz}$. In this simulation and experiment, the amplitude of sinusoidal signal input is $52 \mathrm{~m} V_{p-p}$.

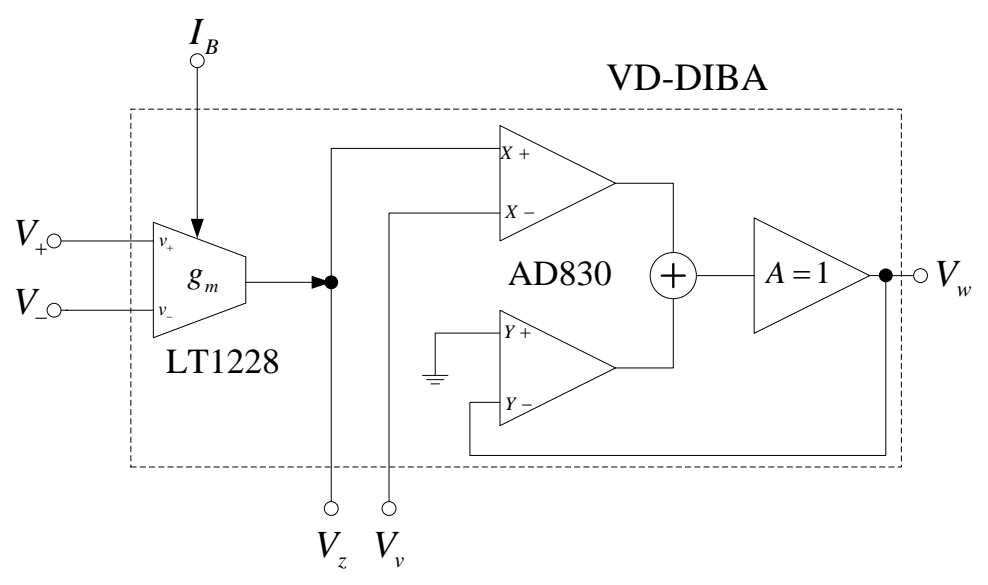

Figure 4. The VD-DIBA implementation by ICs LT1228 and AD830

The simulated and experimental result of the frequency responses of the proposed electronically controllable filter for $\mathrm{LP} \mathrm{HP} \mathrm{AP}_{\mathrm{N}}$ and $\mathrm{AP}_{\mathrm{P}}$ function as illustrated in Figures 5 to 8, respectively compared its characteristics with the ideal case and with the measurement results. From these results, it is found that the gain-frequency responses of the LP HP AP $\mathrm{N}_{\mathrm{N}}$ and $\mathrm{AP}_{\mathrm{P}}$ function are according to the calculation (3) to (6), respectively with an error of about $5 \%$. The bode plot of the phase response of LP and HP function as shown in Figures 9 and 10, respectively It is found that the phase responses are according to the calculation in (7), (8), respectively. The phase responses of $\mathrm{AP}_{\mathrm{N}}$ and $\mathrm{AP}_{\mathrm{P}}$ functions as shown in Figures 11, 12, respectively. It is found that the phase responses are according to the calculation in (9), (10).

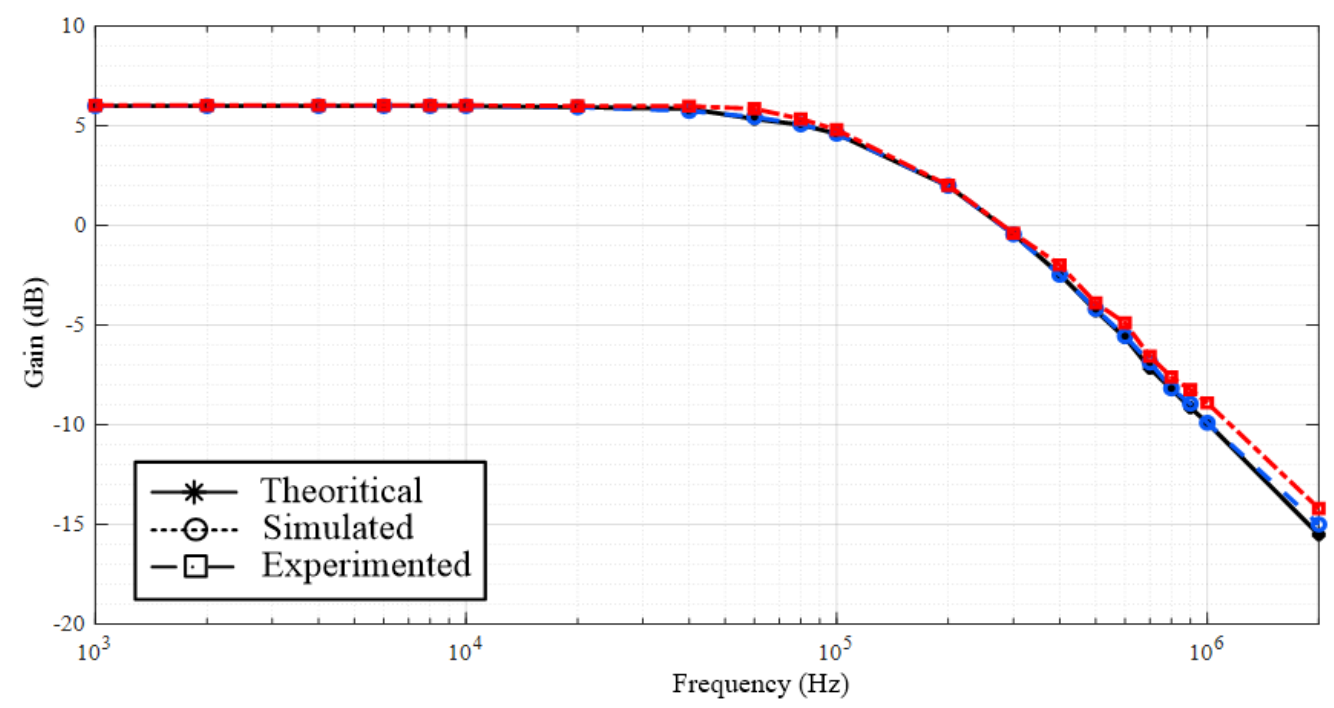

Figure 5. The result of the gain-frequency responses for the LP 


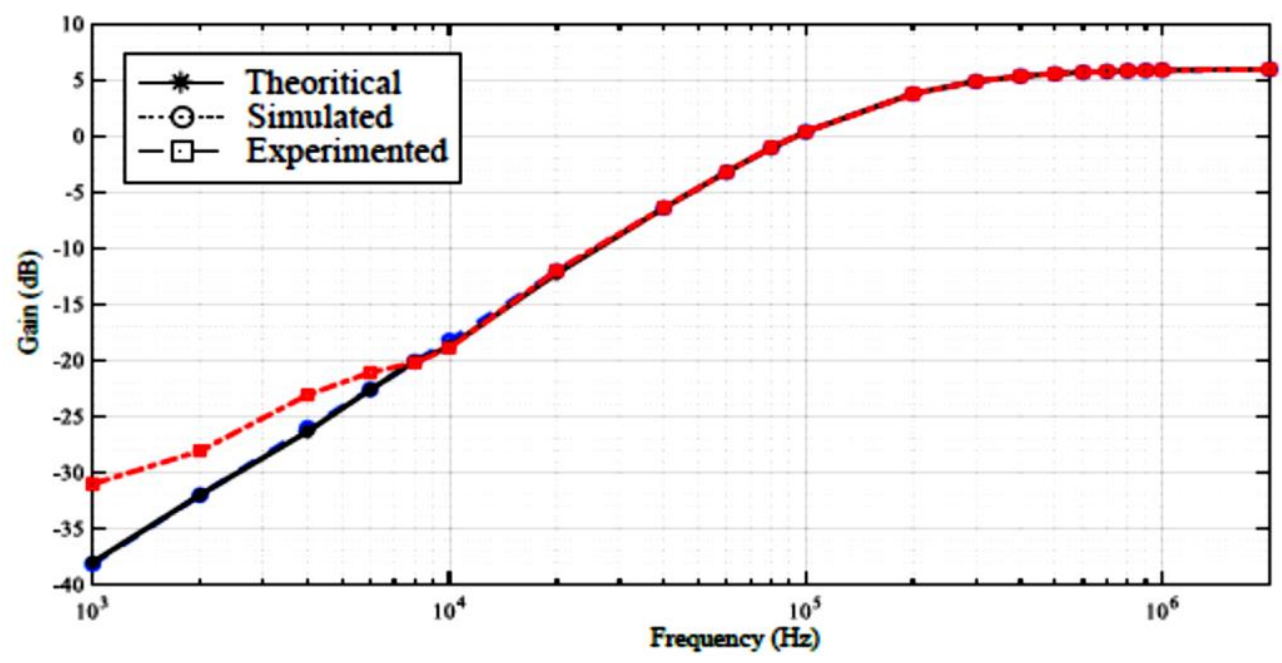

Figure 6. The result of the gain-frequency responses for the HP

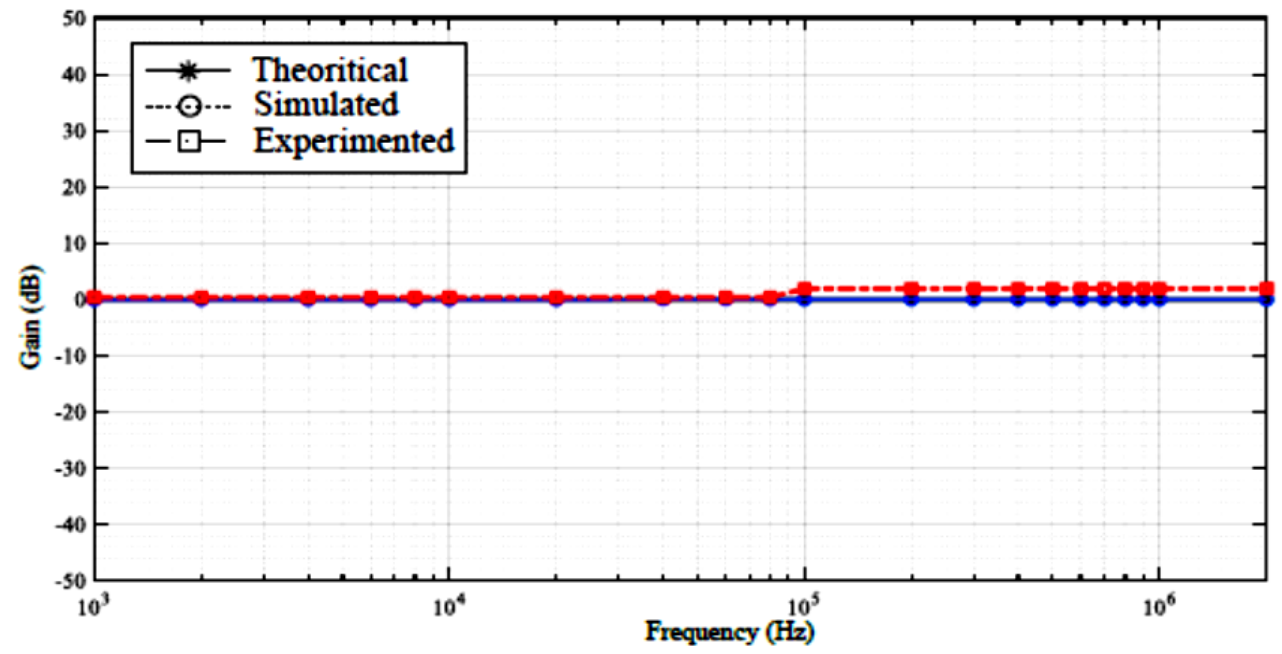

Figure 7. The result of the gain-frequency responses for the $\mathrm{AP}_{\mathrm{N}}$

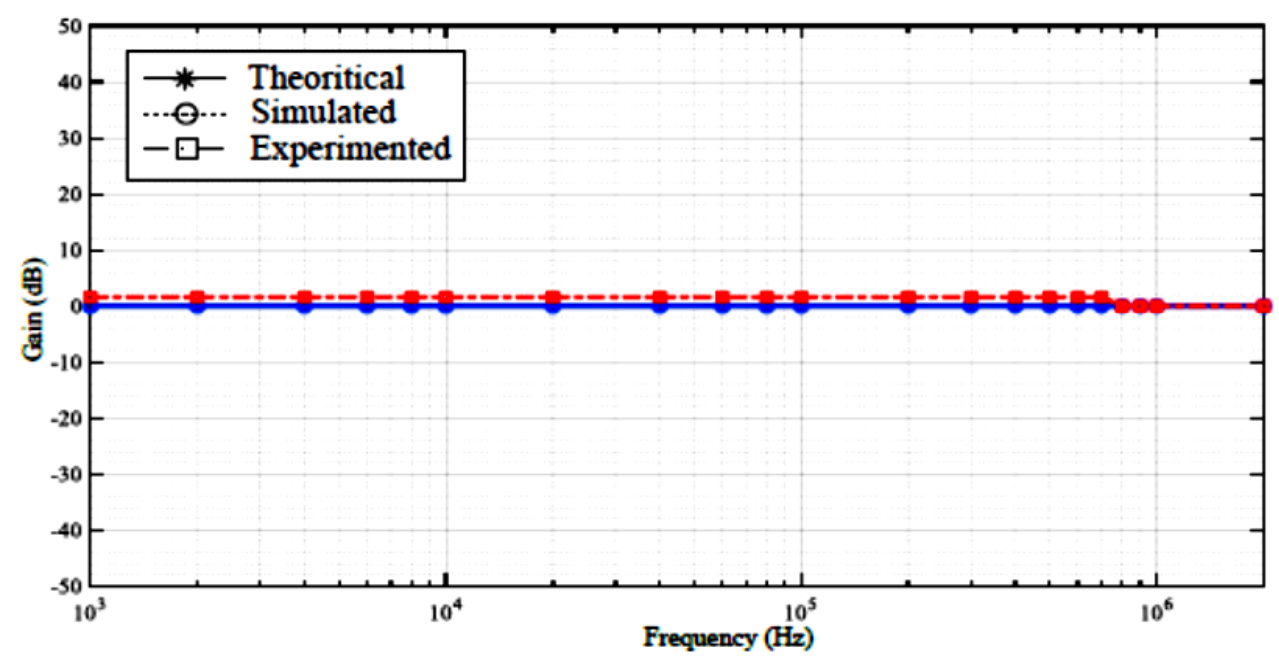

Figure 8. The result of the gain-frequency responses for the APP 


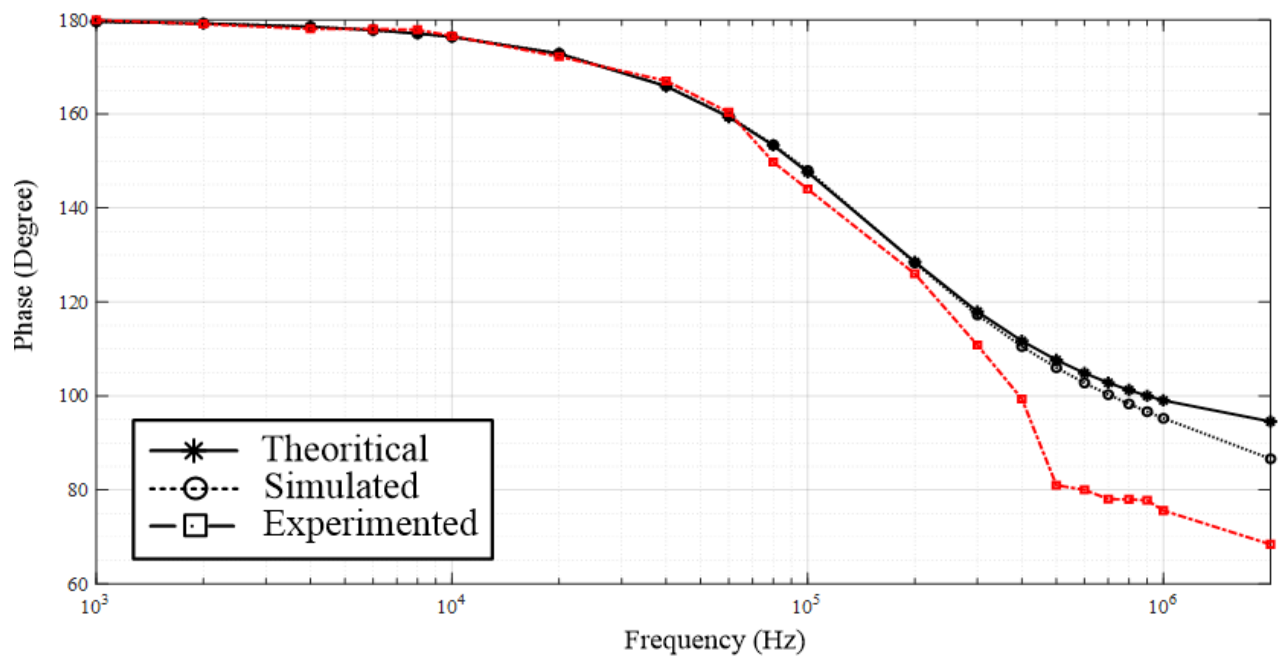

Figure 9. The phase response of the LP

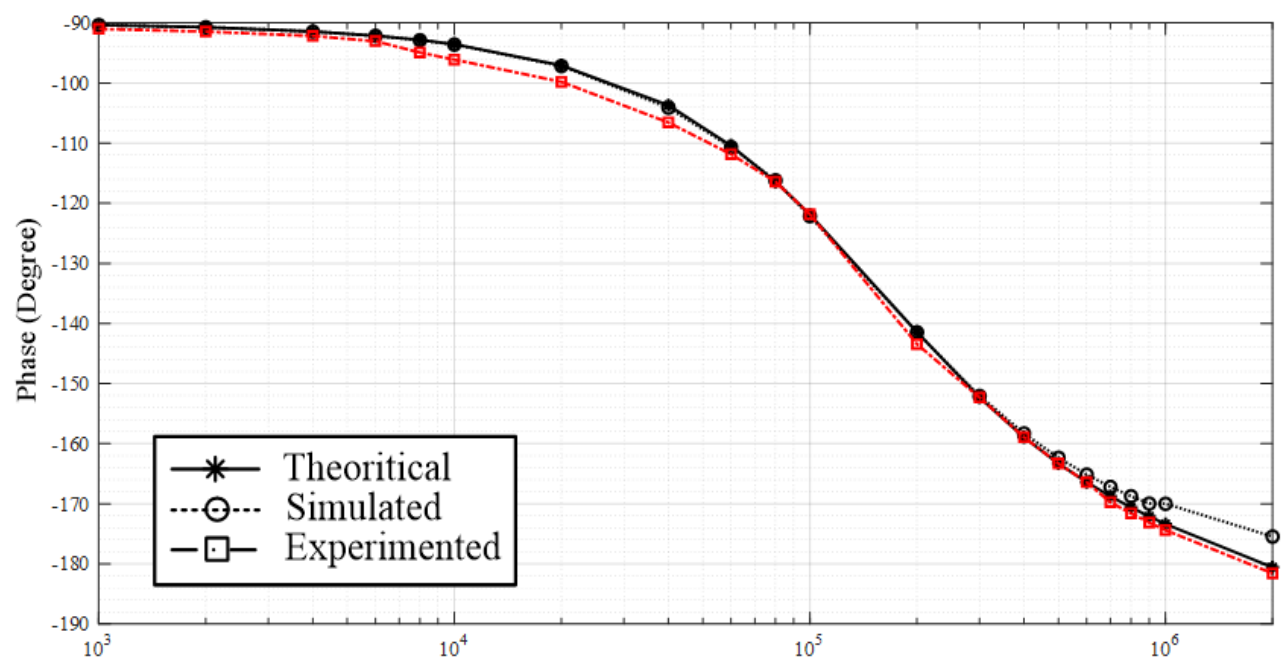

Figure 10. The phase response of the HP

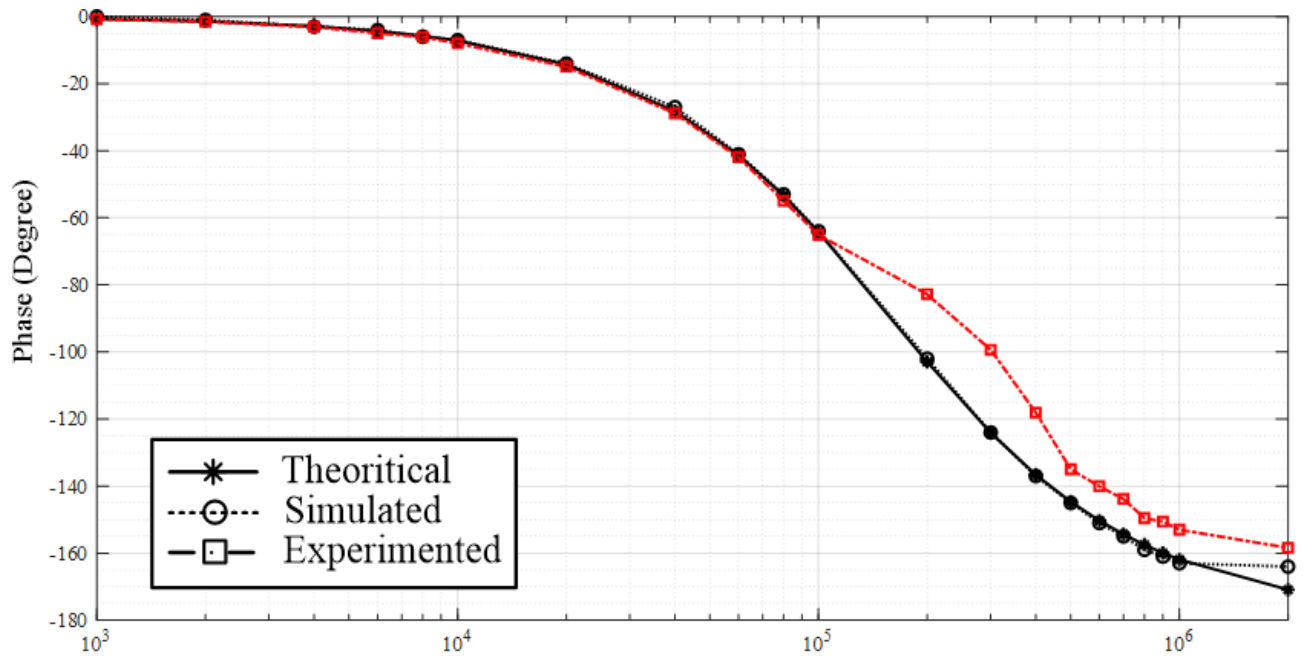

Figure 11. The phase response of the $\mathrm{AP}_{\mathrm{N}}$ 


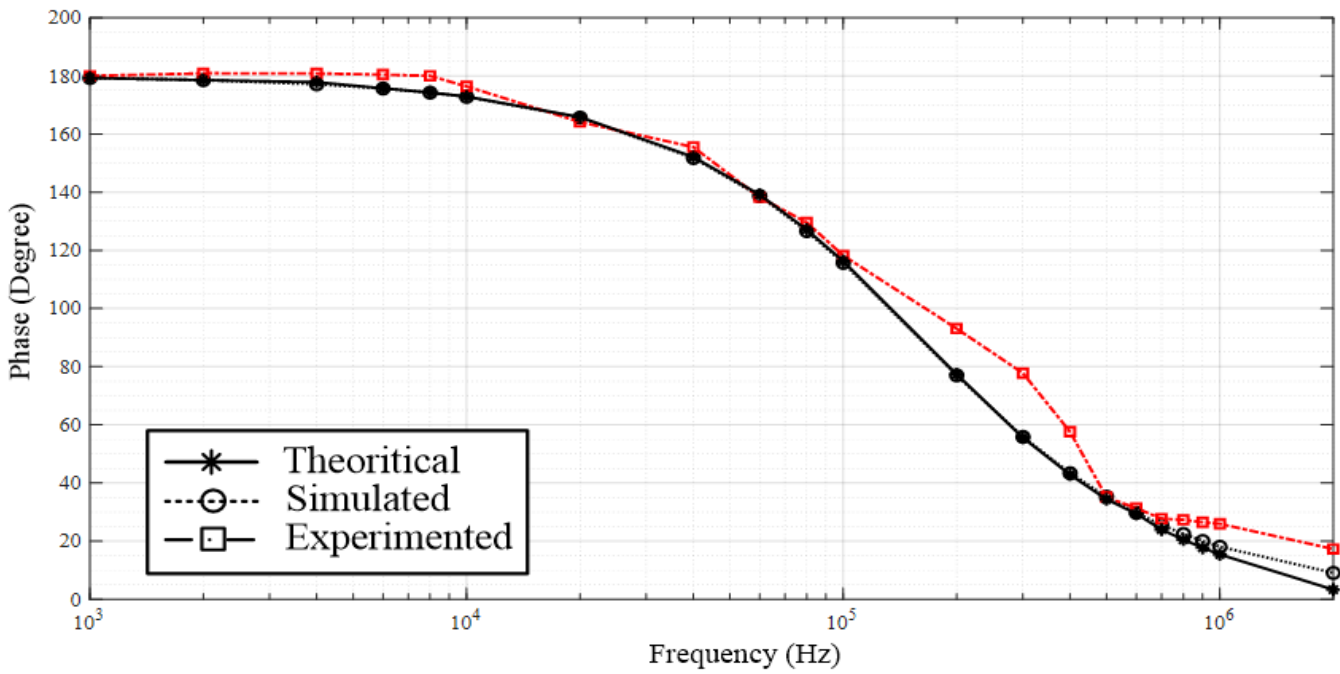

Figure 12. The phase response of the $\mathrm{AP}_{\mathrm{P}}$

Figure 13 illustrates the simulated of the proposed filter for the percentage of total harmonic distortion (\% THD) of the output voltage signal, when entering the sine wave $100 \mathrm{kHz}$ to the input and tune input voltage $5-100 \mathrm{mV}$, for the verification of the linearity of the proposed filter. The total harmonic distortion of the proposed filter is a lesser $1 \%$ the input range of the $\mathrm{AP}_{\mathrm{P}}$ and $\mathrm{AP}_{\mathrm{N}}$ approximately $68 \mathrm{mV}$, the $\mathrm{HP}$ approximately $70 \mathrm{mV}$ and the LP approximately $75 \mathrm{mV}$. Figure 14(a) illustrates the simulated phase property of the $\mathrm{AP}_{\mathrm{N}}$ filtering function when the bias current $\left(I_{B}\right)$ was set to $60 \mu \mathrm{A}, 80 \mu \mathrm{A}$, and $100 \mu \mathrm{A}$ are respectively. It can be clearly seen the phase property from simulation agrees well with theoretical expectations. Figure 14(b) shows the experimental phase property of the $\mathrm{AP}_{\mathrm{N}}$ function. Figure 15 illustrates the output waveforms of the $\mathrm{AP}_{\mathrm{N}}$ filtering function when $I_{B}$ was difference value. It clearly shows that the phase of the output waveform can be shifted when a current bias is adjusted. Figure 16(a) illustrates the simulation phase property of the $\mathrm{AP}$ function when bias current $\left(I_{B}\right)$ was set at $60 \mu \mathrm{A}, 80 \mu \mathrm{A}$, and $100 \mu \mathrm{A}$, respectively. It clearly shows that the simulated phase response agrees reasonably well with theoretical. Figure 16(b) illustrates the experimental phase property of the $\mathrm{AP}_{\mathrm{P}}$ filtering function. It clearly shows that the experimental phase response also agrees well with theoretical expectations. Figure 17 shows the output waveforms of the $\mathrm{AP}_{\mathrm{P}}$ function when $I_{B}$ was difference value. It clearly shows that the phase of the output waveform can be shifted when a current bias is adjusted. In these results, the phase responses of the AP functions were obtained to verify the theoretical analysis in (9) and (10).

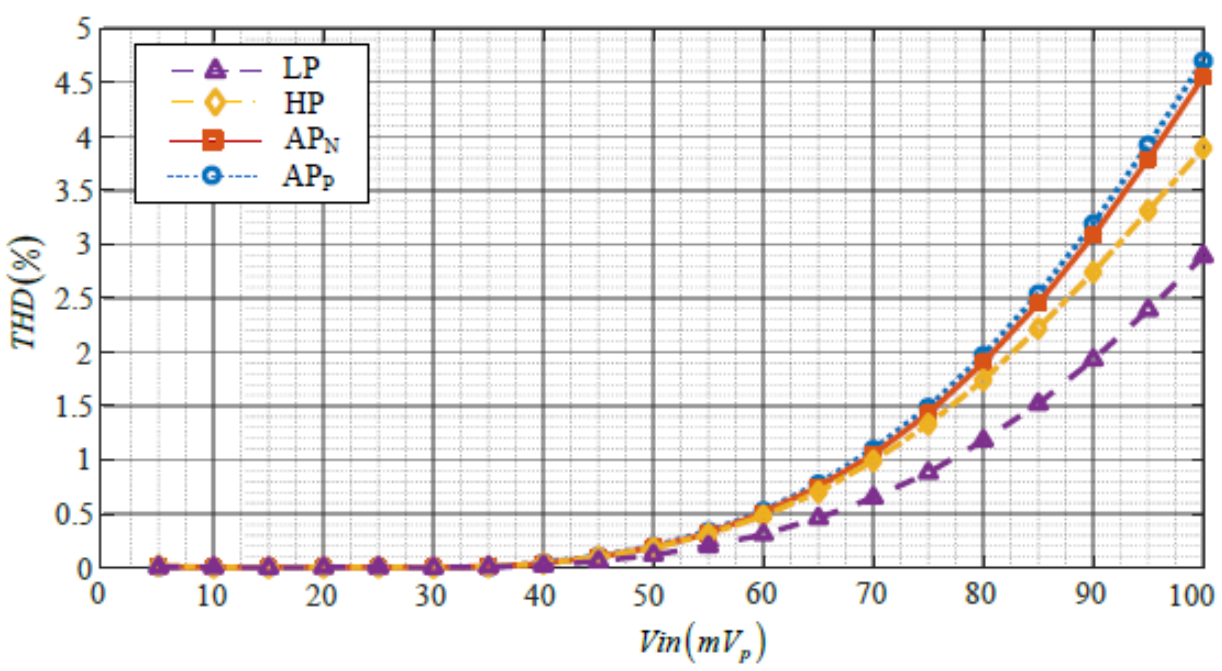

Figure 13. Percent of total harmonic distortion 


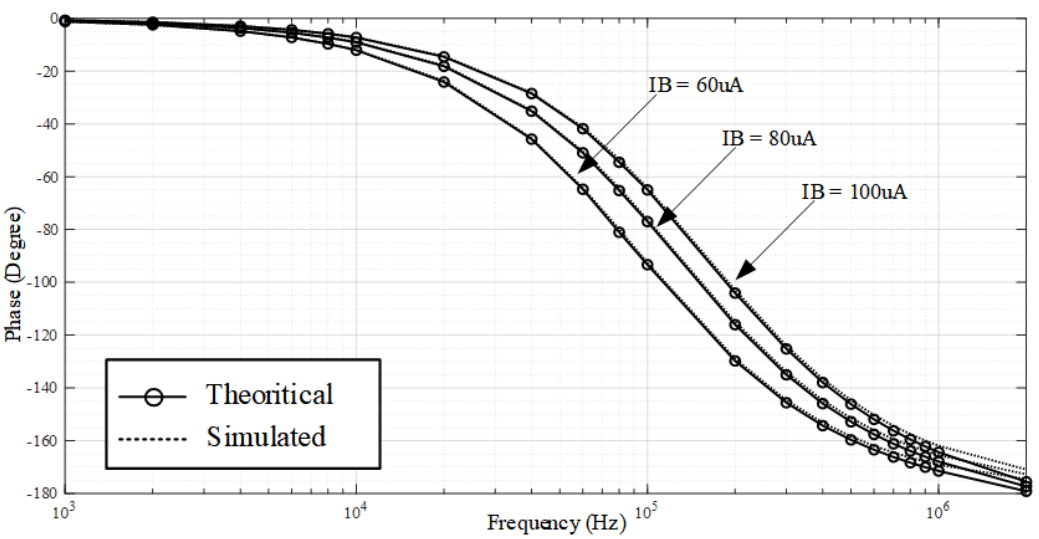

(a)

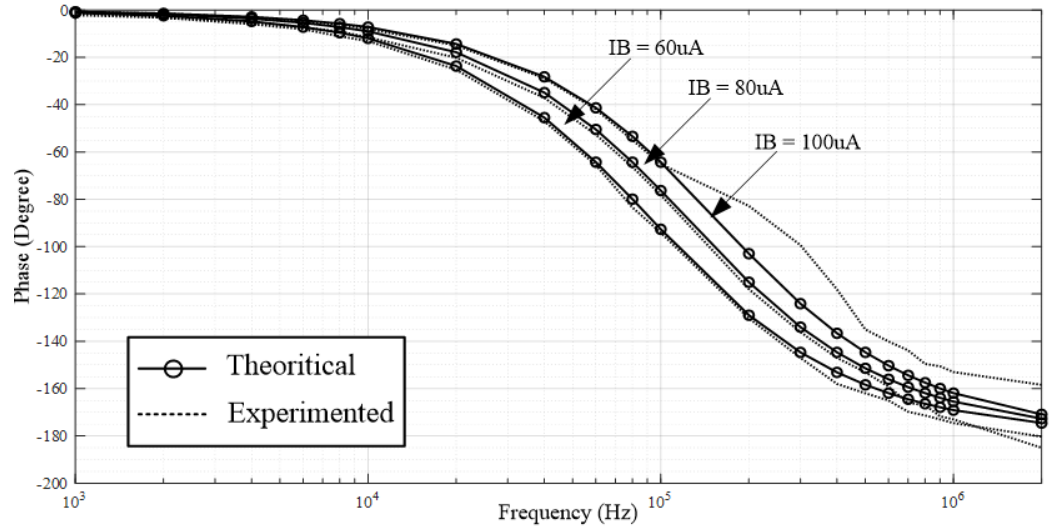

(b)

Figure 14. The phase response of the APN when I_B was difference value, (a) simulations and (b) experimental

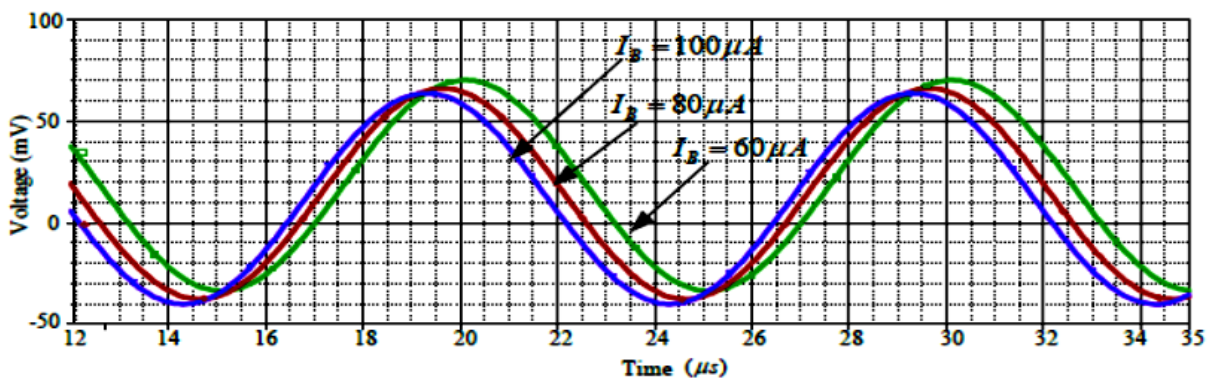

Figure 15. The result of the simulated transient response of the $\mathrm{AP}_{\mathrm{N}}$ when current bias $\left(I_{B}\right)$ are different

This section presents the results of the effect of temperature variance on the efficiency of the proposed filter. Figure 18 shows the result of the simulation of the gain and phase of the APN filter and Figure 19 shows the simulation of the gain and phase of the APP filter when various temperatures 3 values are 0,27 , and $100{ }^{\circ} \mathrm{C}$. Figure 20(a) shows the measured sinusoidal waveforms of input and output signal for the LP function at frequencies $1 \mathrm{kHz}$, the phase response is $180^{\circ}$ degree. Figure 20(b) shows the measured sinusoidal waveforms of input and output signal for the HP function at frequencies $700 \mathrm{kH}_{\mathrm{z}}$, the phase response is $-168.75^{\circ}$. Figures 21 (a) and 21(b) show the measured sinusoidal waveforms of input and output signal for the $\mathrm{AP}_{\mathrm{P}}$ function with frequencies $8 \mathrm{kHz}$ and $800 \mathrm{kHz}$, the phase responses are $127.14^{0}$ and $21^{0}$ respectively. Finally, Figures 21(c)-(d) show the measured sinusoidal waveforms of input and output signal for the $\mathrm{AP}_{\mathrm{N}}$ function with frequencies $8 \mathrm{kHz}$ and $8 \mathrm{kHz}$, the phase responses are $-5.75^{0}$ and $-64.28^{0}$, respectively. 


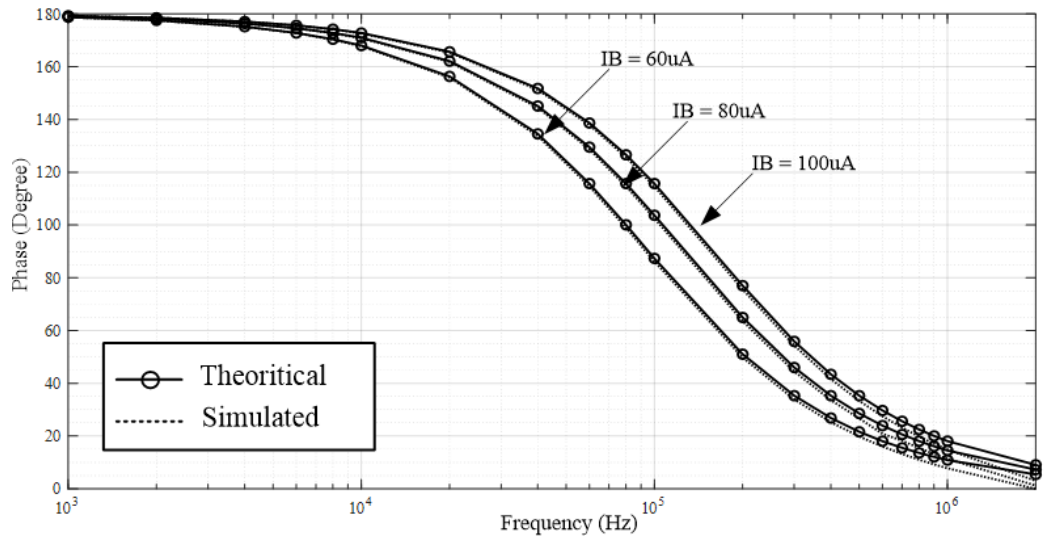

(a)

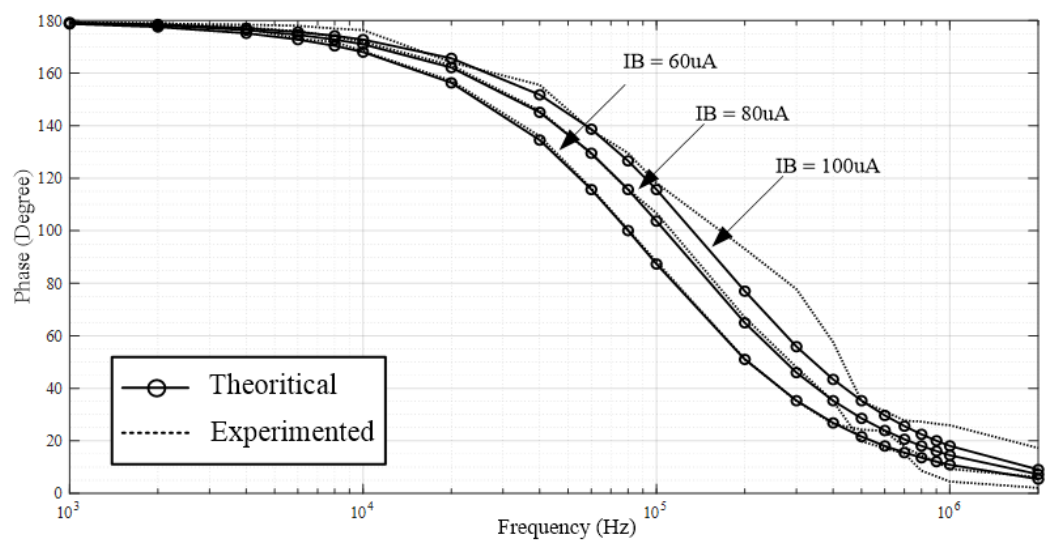

(b)

Figure 16. The phase response of the $\mathrm{AP}_{\mathrm{P}}$ when $I_{B}$ was difference value, (a) simulations and (b) experimental

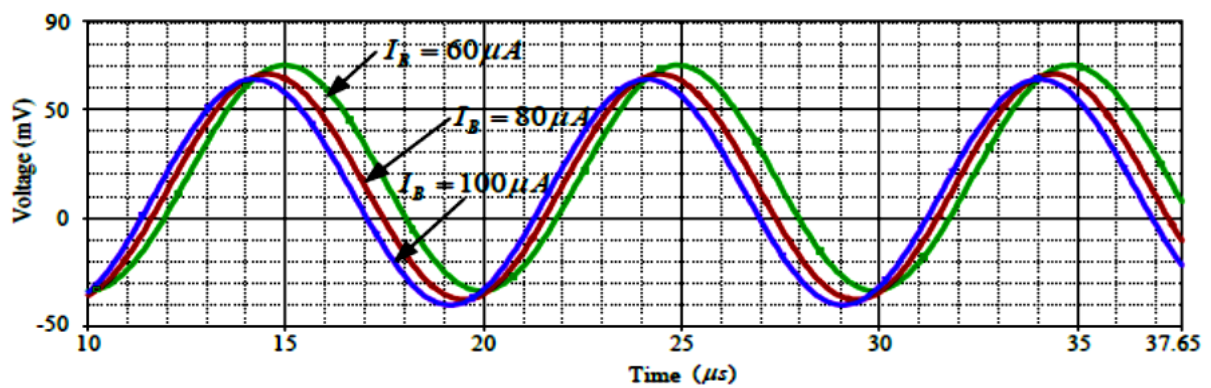

Figure 17. The result of the simulated transient response of the $\operatorname{AP}_{\mathrm{P}}$ when current $\operatorname{bias}\left(I_{B}\right)$ are different

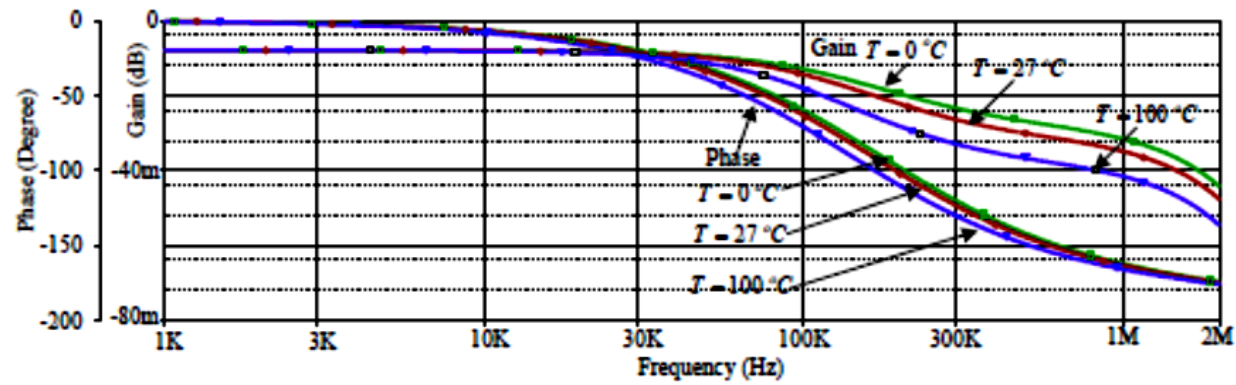

Figure 18. The result of the simulated the gain and phase of the $\mathrm{AP}_{\mathrm{N}}$ when temperatures are different 


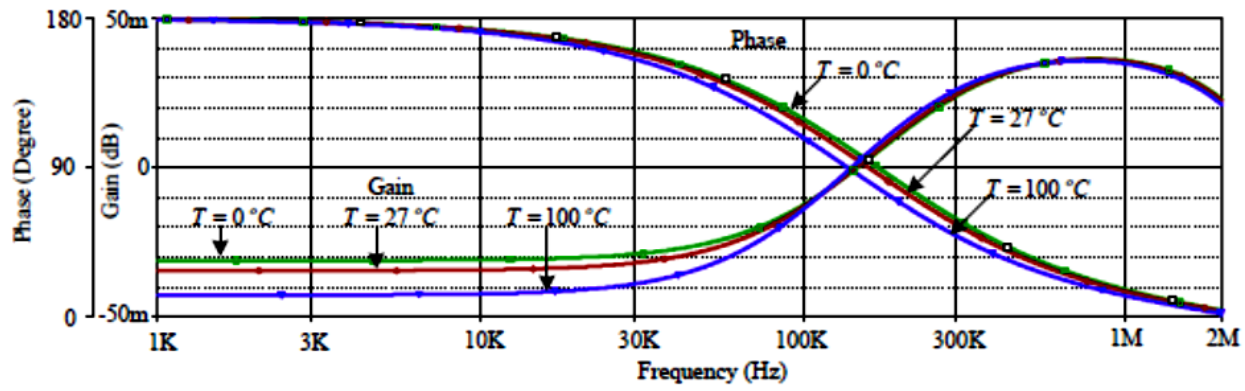

Figure 19. The result of the simulated the gain and phase of the $\mathrm{AP}_{\mathrm{P}}$ when temperatures are different

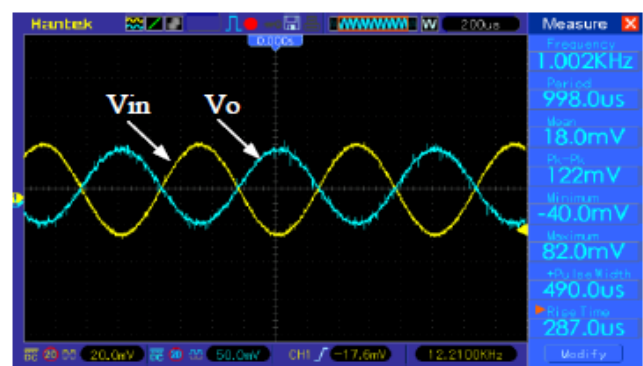

(a)

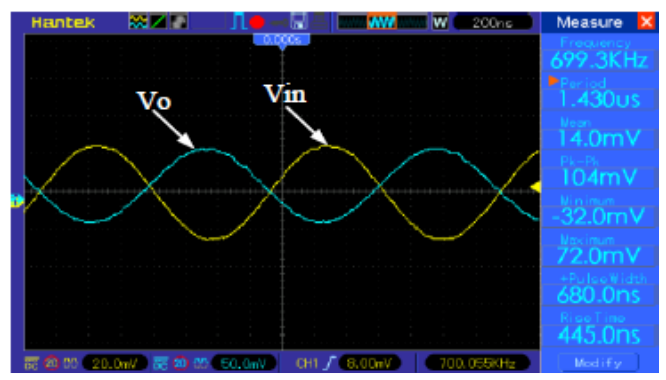

(b)

Figure 20. Measured sinusoidal waveforms $V_{\text {in }}$ and $V_{o}$, (a) LP and (b) HP

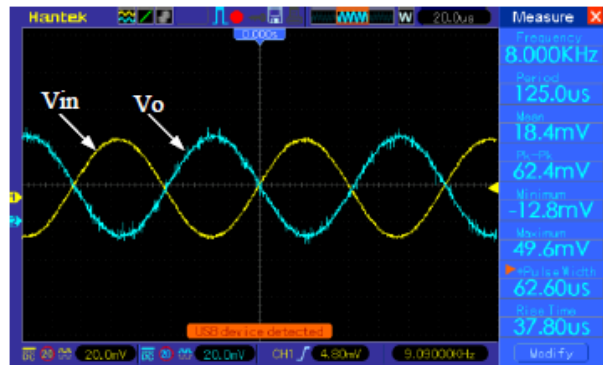

(a)

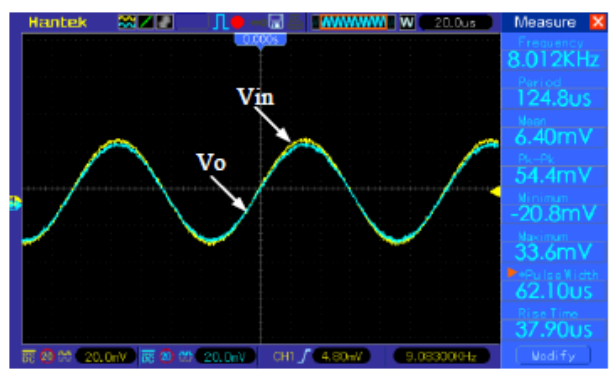

(c)

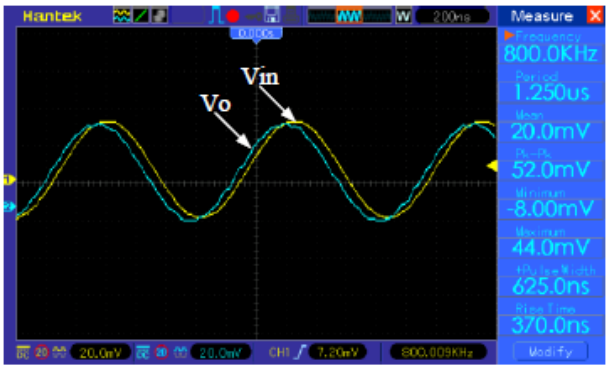

(b)

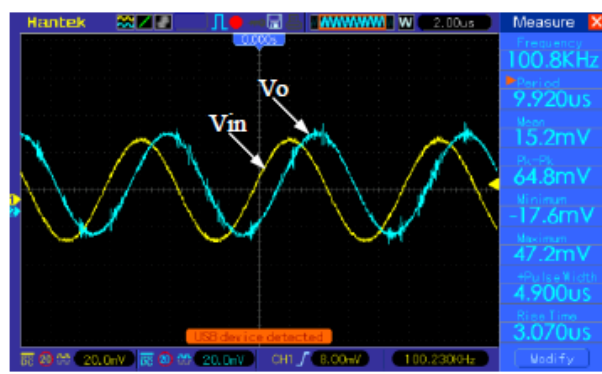

(d)

Figure 21. Measured sinusoidal waveforms $V_{\text {in }}$ and $V_{o}$ of the AP (a) $\mathrm{AP}_{\mathrm{P}}$ with frequencies $8 k H_{z}$, (b) $\mathrm{AP}_{\mathrm{P}}$ with frequencies $800 \mathrm{kH}_{z}$, (c) $\mathrm{AP}_{\mathrm{N}}$ with frequencies $8 k H_{z}$, and (d) $\mathrm{AP}_{\mathrm{N}}$ with frequencies $100 \mathrm{kH}_{z}$

\section{COMPARED WITH UNIVERSAL FIRST-ORDER FILTERS AND APPLICATION}

From the literature survey, the universal first-order filter using active elements and passive elements are has been published. However, the performances of the proposed filters in [7]-[9], [13] contain more than one active building block, lack of electronic controllability [9]-[14], [16], [17], output voltage node is not low impedance [17], and the proposed filters in [7], [8], [17], [9]-[16] are not verified experimentally. 
Table 1 show the results of the proposed filter, in comparison with universal first-order filters, evidence indicates that the proposed filter matches all the standards in the best way among all other filters.

In order to demonstrate the advantages of the proposed universal first-order filter, the second-order band-reject filter (BR) is realized as an application example. The design of the BR is achieved in Figure 22. The BR filter consists of three VD-DIBAs, two capacitors, and five resistors, with three voltage nodes is Vin1, Vin2, and Vo. From the proposed BR in Figure 22, the transfer function in the current-mode form of $\mathrm{BR}$ response can be expressed in (32), the natural frequency can be expressed in (33) and the quality factor can be expressed in (34).

$$
\frac{V_{O}}{V_{\text {in }}}=-2 g_{m 3} R_{L}\left(\frac{s^{2}+\left(\frac{g_{m}}{C}\right)^{2}}{s^{2}+\left(\frac{2 g_{m}}{C}\right) s+\left(\frac{g_{m}}{C}\right)^{2}}\right)
$$

Where $C_{1}=C_{2}=C$ it is found that the voltage gain of the BR can be controlled via $g_{m 3}$ and $R_{L}$.

$$
\omega_{o}=\frac{g_{m}}{C}
$$

It is found that the FO from (33) it can be electronically controlled via $g_{m}$ where $g_{m 1}=g_{m 2}=g_{m}$.

$$
Q=\frac{1}{2}
$$

The workability of the BR filter was verified through the simulation of the PSPICE program. The following parameters were used: the resistor $R_{1}=R_{2}=R_{3}=R_{4}=R=1 \mathrm{k} \Omega$ and $R_{L}=50 \Omega$, the capacitor $C_{1}=C_{2}=C=1 \mathrm{nF}$, the power supply voltages were set at $V_{D D}=-V_{S S}=5 \mathrm{~V}$. We set bias current $\left(I_{B}\right)$ of the VD-DIBA1 and VD-DIBA2 are $100 \mu A$ and the VD-DIBA3 set bias current is $60 \mu A$. Figure 23 shown the simulated of BR gain and phase response, the natural frequency $\left(f_{o}\right)$ is $160 \mathrm{kHz}$, and quality factor are $Q=0.5$. Figure 24 shown the simulated gain were controlled by the bias current $\left(I_{B 3}\right)$ three values are $60 \mu A, 100 \mu A$ and $150 \mu A$. It clearly shows that the simulated gain and phase response agrees reasonably well with theoretical.

Table 1. The compared with universal first-order filters

\begin{tabular}{ccccc}
\hline Reference & Numbers of an active element & Numbers of R+C & Electronic tune & Experiment \\
\hline$[7]$ & Two OTAs & $1+1$ & Yes & No \\
{$[8]$} & Two OTAs & $4+1$ & Yes & No \\
{$[9]$} & Two CCIIs & $4+1$ & No & No \\
{$[10]$} & Sigle CCII & 2 (grouded)+1 & No & No \\
{$[11]$} & Sigle DVCC & $1+1$ & No & No \\
{$[12]$} & Sigle DVCC & $2+1$ & No & No \\
{$[13]$} & Two DVCCs & $1+1$ (grouded) & No & No \\
{$[14]$} & Sigle DDCC & $1+1$ (grouded) & No & No \\
{$[15]$} & Sigle DDCCTA & $0+1$ & Yes & No \\
{$[16]$} & Sigle FDCCII & 1 (grouded)+1(grouded) & No & No \\
{$[17]$} & Sigle VDTA & $0+1$ & No & No \\
The proposed & Sigle VD-DIBA & $2+1$ & Yes & Yes \\
\hline
\end{tabular}

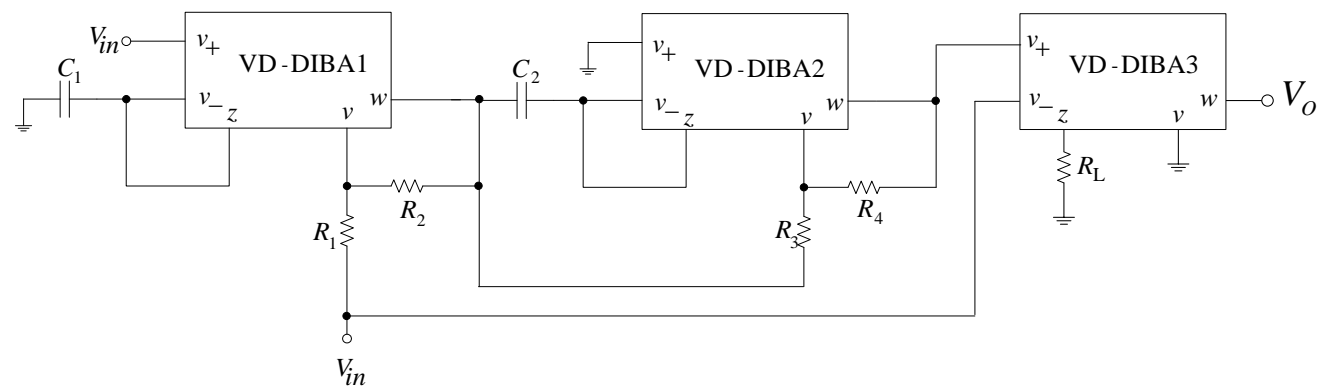

Figure 22. The proposed BR filter 


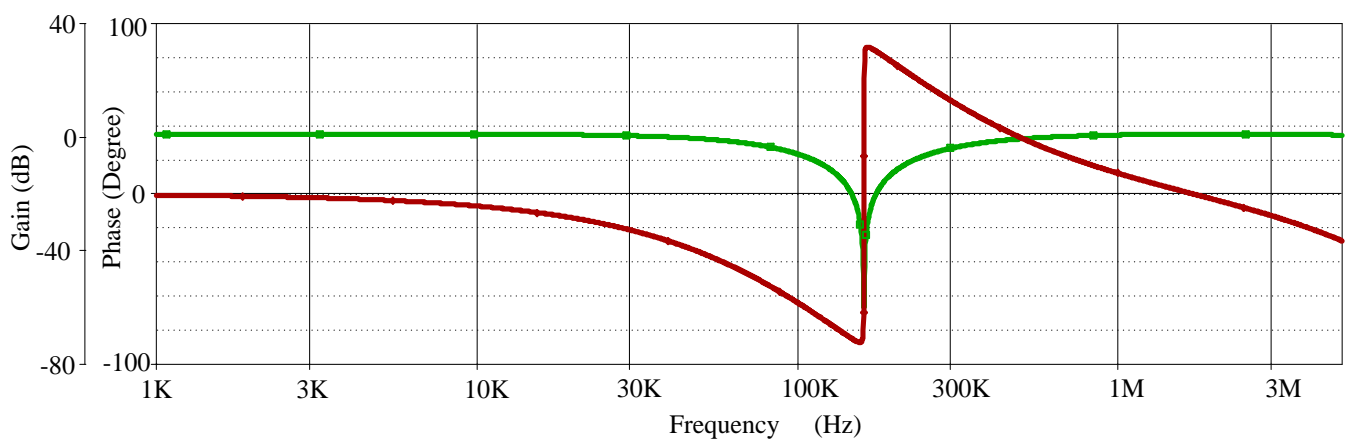

Figure 23. The result of the simulated gain and phase of the BR function

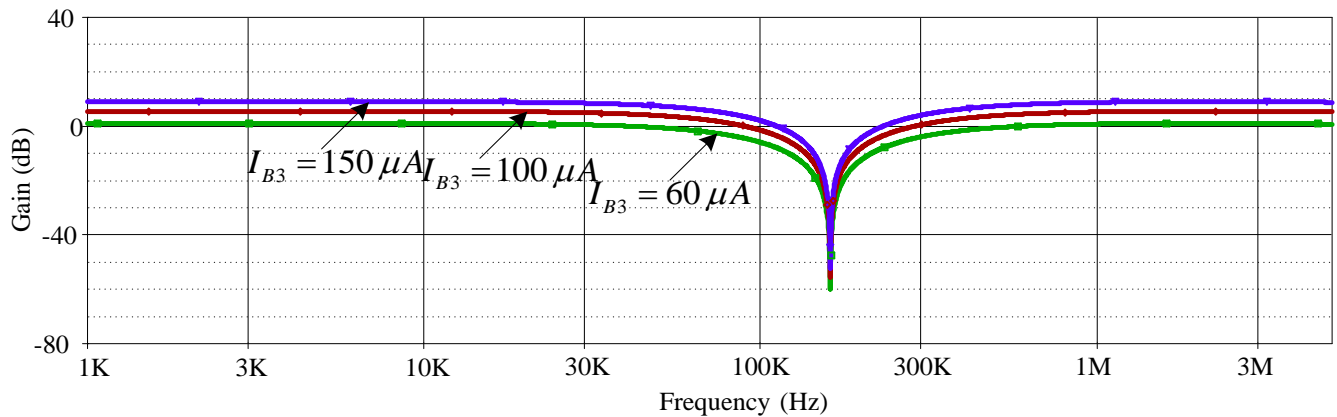

Figure 24. The result of the simulated gain of the BR function when $I_{B 3}$ difference

\section{CONCLUSION}

In this paper, the first-order filter using a single VD-DIBA consisted of two commercial ICs: the operational transconductance amplifier, IC number LT1228, and the differential-input buffer, IC number AD830, two resistors, and a capacitor is presented. The proposed electronically controllable first order filter can provide three voltage-mode first-order functions, all-pass (AP), high-pass (HP) and low-pass (LP) functions. The natural frequency $\left(\omega_{0}\right)$ of the proposed first-order configuration can be electronically controlled by changing the DC bias current. The voltage gain of the LP and HP filters can be controllable. The phase response of an AP configurations can be varied from $0^{\circ}$ to $-180^{\circ}$ and $180^{\circ}$ to $0^{\circ}$. The bandwidth of the proposed filter is $28.81 \mathrm{MHz}$. The power supply voltages were set at $\pm 5 \mathrm{~V}$. Verification of the theoretically described performances of the presented electronically tunable first order universal filter was proved by the PSpice simulations and experiment.

\section{REFERENCES}

[1] C. Psychalinos, C. Kasimis, and F. Khateb, "Multiple-input single-output universal biquad filter using single output operational transconductance amplifiers," AEU - International Journal of Electronics and Communications, vol. 93, pp. 360-367, Sep. 2018, doi: 10.1016/j.aeue.2018.06.037.

[2] M. A. Ibrahim, S. Minaei, and H. Kuntman, "A $22.5 \mathrm{MHz}$ current-mode KHN-biquad using differential voltage current conveyor and grounded passive elements," AEU - International Journal of Electronics and Communications, vol. 59, no. 5, pp. 311-318, Jul. 2005, doi: 10.1016/j.aeue.2004.11.027.

[3] K. L. Pushkar, D. R. Bhaskar, and D. Prasad, "Voltage-mode universal biquad filter employing single voltage differencing differential input buffered amplifier," Circuits and Systems, vol. 4, no. 1, pp. 44-48, 2013, doi: 10.4236/cs.2013.41008.

[4] W. Jaikla et al., "Synthesis of biquad filters using two VD-DIBAs with independent control of quality factor and natural frequency," AEU - International Journal of Electronics and Communications, vol. 132, p. 153601, Apr. 2021, doi: 10.1016/j.aeue.2020.153601.

[5] W. Jaikla, F. Khateb, T. Kulej, and K. Pitaksuttayaprot, "Universal filter based on compact cmos structure of vddda," Sensors, vol. 21, no. 5, pp. 1-25, Mar. 2021, doi: 10.3390/s21051683.

[6] W. Jaikla, F. Khateb, M. Kumngern, T. Kulej, R. K. Ranjan, and P. Suwanjan, "0.5 V fully differential universal filter based on multiple input OTAs," IEEE Access, vol. 8, pp. 187832-187839, 2020, doi: 10.1109/ACCESS.2020.3030239.

[7] M. Gupta, T. S. Arora, and S. N. Gupta, "A novel current-mode and voltage-mode all pass filter employing operational transconductance amplifier," in 2016 IEEE 1st International Conference on Power Electronics, Intelligent Control and Energy Systems (ICPEICES), Jul. 2016, pp. 1-3, doi: 10.1109/ICPEICES.2016.7853058.

[8] W. Jaikla, P. Talabthong, S. Siripongdee, P. Supavarasuwat, P. Suwanjan, and A. Chaichana, "Electronically controlled voltage mode first order multifunction filter using low-voltage low-power bulk-driven OTAs," Microelectronics Journal, vol. 91, 
pp. 22-35, Sep. 2019, doi: 10.1016/j.mejo.2019.07.009.

[9] N. A. Shah, S. Z. Iqbal, and B. Parveen, "Simple first-order multifunction filter," Indian Journal of Pure and Applied Physics, vol. 42, no. 11, pp. 854-856, 2004.

[10] I. A. Khan and S. Maheshwari, "Simple first order all-pass section using a single CCII," International Journal of Electronics, vol. 87, no. 3, pp. 303-306, Mar. 2000, doi: 10.1080/002072100132200.

[11] H. P. Chen, S. F. Wang, and K. W. Huang, "Grounded-capacitor first-order filter employing single DVCC," in 2011 IEEE 3rd International Conference on Communication Software and Networks, ICCSN 2011, May 2011, pp. 401-404, doi: 10.1109/ICCSN.2011.6014922.

[12] J. W. Horng, "High input impedance first-order allpass, highpass and lowpass filters with grounded capacitor using single DVCC," Indian Journal of Engineering and Materials Sciences, vol. 17, no. 3, pp. 175-178, 2010.

[13] J. W. Horng, "DVCCs based high input impedance voltage-mode first-order allpass, highpass and lowpass filters employing grounded capacitor and resistor," Radioengineering, vol. 19, no. 4, pp. 653-656, Dec. 2010

[14] J. W. Horng, C. L. Hou, C. M. Chang, Y. T. Lin, I. C. Shiu, and W. Y. Chiu, "First-order allpass filter and sinusoidal oscillators using DDCCs," International Journal of Electronics, vol. 93, no. 7, pp. 457-466, Jul. 2006, doi: 10.1080/00207210600711481.

[15] M. Kumngern, V. Mongkol, and S. Junnapiya, "Voltage-mode allpass section employing only one DDCCTA and one capacitor," in 2013 Eleventh International Conference on ICT and Knowledge Engineering, Nov. 2013, pp. 1-4, doi: 10.1109/ICTKE.2013.6756279.

[16] B. Metin, N. Herencsar, and K. Pal, "Supplementary first-order all-pass filters with two grounded passive elements using FDCCII," Radioengineering, vol. 20, no. 2, pp. 433-437, 2011.

[17] S. Maneewan, N. Udorn, D. Duangmalai, P. Silapan, and W. Jaikla, "A voltage-mode first order allpass filter based on VDTA," Advances in Electrical and Electronic Engineering, vol. 12, no. 1, pp. 40-46, Mar. 2014, doi: 10.15598/aeee.v12i1.846.

[18] D. Biolek and V. Biolkova, "First-order voltage-mode all-pass filter employing one active element and one grounded capacitor," Analog Integrated Circuits and Signal Processing, vol. 65, no. 1, pp. 123-129, Dec. 2010, doi: 10.1007/s10470-009-9435-2.

[19] W. Jaikla, D. Biolek, S. Siripongdee, and J. Bajer, "High input impedance voltage-mode biquad filter using VD-DIBAs," Radioengineering, vol. 23, no. 3, pp. 914-921, 2014

[20] K. L. Pushkar, D. R. Bhaskar, and D. Prasad, "Single-resistance-controlled sinusoidal oscillator using single VD-DIBA," Active and Passive Electronic Components, vol. 2013, pp. 1-5, 2013, doi: 10.1155/2013/971936.

[21] W. Ninsraku, D. Biolek, W. Jaikla, S. Siripongdee, and P. Suwanjan, "Electronically controlled high input and low output impedance voltage mode multifunction filter with grounded capacitors," AEU - International Journal of Electronics and Communications, vol. 68, no. 12, pp. 1239-1246, Dec. 2014, doi: 10.1016/j.aeue.2014.07.004.

[22] P. Jardrit, W. Jaikla, S. Siripogdee, A. Chaichana, R. Sotner, and F. Khateb, "Design of high input impedance voltage-mode multifunction biquad filter with independent control of natural frequency and quality factor," in 17th International Conference on Electrical Engineering/Electronics, Computer, Telecommunications and Information Technology, ECTI-CON 2020, Jun. 2020, pp. 234-237, doi: 10.1109/ECTI-CON49241.2020.9158266.

[23] W. Jaikla, S. Bunrueangsak, F. Khateb, T. Kulej, P. Suwanjan, and P. Supavarasuwat, "Inductance simulators and their application to the 4th order elliptic lowpass ladder filter using cmos vd-dibas," Electronics, vol. 10, no. 6, p. 684, Mar. 2021, doi: 10.3390/electronics10060684.

[24] Linear Technology Corporation, "LT1228 - 100MHz current feedback amplifier with DC gain control," Linear Technology Corporation, Accessed: Sep. 09, 2021. [Online]. Available: https://www.analog.com/media/en/technical-documentation/datasheets/1228fd.pdf

[25] Microchip Technology Inc. "AD830, high speed, video difference amplifier,” Microchip Technology Inc., Accessed: Sep. 09, 2021. [Online]. Available: https://www.analog.com/media/en/technical-documentation/data-sheets/ad830.pdf 